\title{
Auf verlorenem Posten? Eine Untersuchung zu Einflussmöglichkeiten der Opposition im Bayerischen Landtag
}

\author{
Katrin Steinack
}

Die politischen Besonderheiten Bayerns sind in den vergangenen Jahrzehnten vielfältig hervorgehoben und untersucht worden. Unter dem Stichwort „Bayerns Uhren gehen anders“ wurde über die spezifische Politische Kultur des Freistaats diskutiert ${ }^{1}$, es wurden die Auswirkungen der hegemonialen Rolle der „bayerischen Staatspartei“ CSU auf den Parteienwettbewerb innerhalb des Flächenstaates begutachtet ${ }^{2}$, und die Besonderheiten des bayerischen Landtagswahlrechts wurden neben einer politikwissenschaftlichen Analyse auch vor dem Bayerischen Verfassungsgerichtshof einer Prüfung unterzogen. ${ }^{3}$ Zuletzt war die herausragende Stellung des bayerischen Ministerpräsidenten, der im Gegensatz zu seinen Kollegen in den anderen Bundesländern zwar aus eigenem Antrieb zurücktreten, jedoch durch kein parlamentarisches Misstrauensvotum gestürzt werden kann, wiederholt betont worden. ${ }^{4}$

In keinem anderen deutschen Landesparlament scheinen die Mehrheiten so klar verteilt und dauerhaft zementiert wie im Bayerischen Landtag. Die SPD befindet sich seit 1957 in der Opposition und hält damit einen europaweit gültigen Rekord parlamentarischer Niederlagen. ${ }^{5}$ Die absolute Mehrheit der CSU in Bayern ist seit 1962 unangefochten, so dass diese nicht auf die Bildung einer Regierungskoalition angewiesen ist. FDP und Bündnis 90/ Die Grünen als kleinere Oppositionsparteien waren in der Vergangenheit nur unregelmäßig und mit einer geringen Zahl von Mandaten im Landtag vertreten.

1 Vgl. Jürgen W. Falter, Bayerns Uhren gehen wirklich anders. Politische Verhaltens- und Einstellungsunterschiede zwischen Bayern und dem Rest der Republik, in: ZParl, 13. Jg. (1982), H. 4, S. 504 - 521; Jürgen Gebhard, Bayern, Deutschlands eigenwilliger Freistaat. Historisch-gesellschaftliche Aspekte der politischen Kultur in Bayern, in: Rainer A. Roth (Hrsg.), Freistaat Bayern. Die politische Wirklichkeit eines Landes in der Bundesrepublik Deutschland, 4. Auflage, München 1986, S. 83 - 104; Alf Mintzel, Gehen Bayerns Uhren wirklich anders?, in: ZParl, 18. Jg. (1987), H. 1, S. 77 - 93; Jürgen W. Falter, Wie gehen sie denn nun wirklich, die bayerischen Uhren?, in: ZParl, 19. Jg. (1988), H. 1, S. 113 f.

2 Vgl. Alf Mintzel, Die CSU-Hegemonie in Bayern. Strategie und Erfolg, Gewinner und Verlierer, Passau 1998.

3 Zu den Besonderheiten der Wahlen in Bayern auf Landesebene vgl. Emil Hübner, Das bayerische Landtagswahlrecht, in: Reinhold L. Bocklet (Hrsg.), Das Regierungssystem des Freistaates Bayern, Bd. 2, München 1979, S. 279 - 294. Zur Klage vor dem BayVerfG siehe Josef Aulehner, Die Disproportion von Stimmen- und Mandatsanteilen in der Bayerischen Landtagswahl, in: Bayerische Verwaltungsblätter, 19. Jg. (1991), S. 577 - 584 sowie Rainer-Olaf Schultze / Jürgen Ender, Aus aktuellem Anlaß: Bayerns Wahlsystem - verfassungspolitisch bedenklich?, in: ZParl, 22. Jg. (1991), H. 1, S. $150-160$.

4 Vgl. unter anderem Siegfried Mielke, Länderparlamentarismus, Bonn 1971, S. 15; Heinz Rausch, Die Bayerische Staatsregierung, in: Reinhold L. Bocklet (Hrsg.), Das Regierungssystem des Freistaates Bayern, Bd. 1, München 1977, S. 395 - 429, S. 396 f.; Rainer-Olaf Schultze / Jürgen Ender, a.a.O. (Fn. 3), S. 154.

5 Vgl. Warum bekommt die SPD keinen Fuß auf diesen schönen Boden?, in: SZ Magazin vom 19. September 2003. 
Die lange Regierungszeit einer einzigen Partei, die ihre politischen Vorstellungen seit mehr als vier Dekaden ungehindert von Koalitionszwängen umsetzen kann, ist in der Bundesrepublik einmalig. ${ }^{6}$ Die hegemoniale Stellung der Christlichsozialen Union, die in Bayern (im Gegensatz zu den Landesverbänden der anderen Parteien) als Bundespartei antritt, scheint auf unabsehbare Zeit ungefährdet und hat sich mit der Landtagswahl 2003, bei der die Partei erstmals die Zweidrittelmehrheit erlangte, weiter manifestiert. ${ }^{7}$ Angesichts der Überzahl an CSU-Abgeordneten sowie des mächtigen Verwaltungsapparats der Staatskanzlei und der Ministerialverwaltungen wirken die Sozialdemokraten ${ }^{8}$, aber auch die Vertreter kleinerer Oppositionsfraktionen im Landtag den Entscheidungen der Mehrheit ohne Perspektive auf einen baldigen Wechsel nahezu hilflos ausgeliefert. Bei ihren Vorsitzenden führt dies zu Klagen über die „Bedrohung des Parlamentarismus“ sowie zu Einschätzungen wie dieser: „Das Parlament existiert als solches nicht, wir haben die Opposition und die Hilfstruppen der Regierung“, aber „die wirkliche Diskussion (...) wird in den außer- und vorparlamentarischen Raum verlagert" . Dies entspricht den Forschungsergebnissen Samy Gleitmanns, der bereits für die frühen 1970er Jahre die Bedeutungslosigkeit der Opposition im Maximilianeum wie folgt beurteilte:

„Der Opposition, als dem in der Öffentlichkeit auftretenden Widerpart von Regierung und Mehrheit, kommt nach den Erfolgschancen ihrer Initiativen eine völlig untergeordnete, ja fast belanglose Rolle bei der Gesetzgebung zu (...). [Damit] konnte die Opposition ihren Anspruch als ,anderer Beweger der Politik' nur ungenügend erfüllen. Gerade das für das Funktionieren einer parlamentarischen Demokratie so wichtige Wechselspiel zwischen Regierung, Mehrheitsfraktion und Opposition scheint in Bayern nicht zu funktionieren." 10

Die fast ein halbes Jahrhundert währende Dominanz der CSU wirft die Frage auf, inwieweit gängige Forschungshypothesen zur parlamentarischen Opposition, die in der Ver-

6 Vor allem in der Frühphase der Bundesrepublik waren Koalitionsregierungen auf Landesebene die Regel. Vgl. zu den Regierungskonstellationen in den Ländern Peter Schindler, Datenhandbuch zur Geschichte des Deutschen Bundestages 1949-1999. Gesamtausgabe in drei Bänden, Baden-Baden 1999, Bd. I, S. 1439 ff.

7 Die CSU hatte mit 60,7 Prozent der Stimmen 124 Sitze im mit Beginn der 15. Legislaturperiode auf 180 Sitze verkleinerten Landtag erhalten. Während Bündnis 90/Die Grünen sich mit 7,7 Prozent der Stimmen und 15 Mandaten gegenüber vergangenen Legislaturperioden deutlich verbessert hatten, hatte die SPD mit nur 19,6 Prozent der Stimmen und 41 Sitzen das bislang schlechteste Ergebnis ihrer Geschichte erzielt. Vgl. dazu Günter Rieger, Die bayerische Landtagswahl vom 21. September 2003: Banale Sensationen, in: ZParl, 34. Jg. (2003), H. 4, S. $702-$ 720 .

8 Mit der männlichen Form „Sozialdemokraten“, „Politiker“, „Parlamentarier“, „Bürger“ etc. sind im Folgenden - soweit nicht explizit anders benannt - immer auch die weiblichen Parteimitglieder, Abgeordneten, Politikerinnen, Bürgerinnen etc. gemeint.

9 So die Einschätzungen von Franz Maget (SPD) und Sepp Dürr (Bündnis 90/Die Grünen), vgl. Das Parlament erstarrt immer öfter in einer selbstgewählten Ohnmacht. Niedergang des hohen Hauses, in: Süddeutsche Zeitung vom 30. Juni 2001.

10 Samy Gleitmann, Der Bayerische Landtag. Eine Funktionsanalyse unter besonderer Berücksichtigung der Gesetzgebung in der 7. Wahlperiode (1970-74), Konstanz 1980, S. 137. Zum oft zitierten Bild der Opposition als ,anderer Beweger der Politik“ vgl. Carlo Schmid, Opposition als Staatseinrichtung, in: Hans-Gerd Schumann (Hrsg.), Die Rolle der Opposition in der Bundesrepublik Deutschland, Darmstadt 1976, S. 54 - 65, S. 59 f., erstmals abgedruckt in: Der Wähler, 5. Jg. (1955), S. $498-506$. 
gangenheit vor allem angesichts von Koalitionsregierungen in Bund und Ländern entwickelt worden sind ${ }^{11}$, auch für den Bayerischen Landtag Gültigkeit beanspruchen können.

\section{Untersuchungsfeld und Forschungsstand}

Nach herkömmlicher Meinung ist der Aktionsrahmen der parlamentarischen Opposition durch die Aufgaben Kritik, Kontrolle und Alternative abgesteckt. ${ }^{12}$ Das gemeinhin angenommene Hauptziel der Opposition, die Regierung zu ersetzen, kann auf mehreren Wegen erreicht werden. Ausgehend von der Intention ihrer Träger bilden die verschiedenen, vornehmlich in den 1960er und 1970er Jahren entwickelten Einteilungen oppositioneller Strategien in der Regel eine Trias entlang der Parameter Fundamentalopposition, Konkurrenz zur und Kooperation mit der Regierungsmehrheit. ${ }^{13}$

Sowohl in der Parlamentarismusforschung als auch in der international vergleichenden Parteienforschung und der Elitenforschung überwiegen Thesen, die auf ein kooperatives Verhalten von Abgeordneten der Oppositionsparteien gegenüber den Parlamentariern der Mehrheitspartei hindeuten. ${ }^{14}$ Dabei tragen das konstruktiv-kooperative Verhalten der Opposition gegenüber Regierungsentwürfen sowie der gesellschaftliche Zwang, komplexe Probleme im Konsens zu lösen, häufig zu einer gemeinschaftlichen Regelung von Sachverhalten bei. So sind oft neben der klaren Handschrift der Regierungsmehrheit inhaltlich deutliche Lösungsansätze aus dem oppositionellen Lager erkennbar. Die Opposition kooperiert demnach nicht nur, sondern regiert - so die Ansicht der neueren Forschung regelrecht mit. ${ }^{15}$ Der oppositionelle Trend zur Kooperation ist zuweilen auch in Parlamen-

11 Vgl. dazu Katrin Steinack, Opposition im Bayerischen Landtag 1994-1998. Ebenen und Strategien politischer Einflussnahme in einem hegemonialen System, Frankfurt am Main u.a. 2007, S. $34 \mathrm{ff}$.

12 Zum Dreiklang der Oppositionsfunktionen vgl. Dolf Sternberger, Lebende Verfassung. Studien über Koalition und Opposition, Meisenheim am Glan 1956, S. 134.

13 Otto Kirchheimer, Wandlungen der politischen Opposition, in: Kurt Kluxen (Hrsg.), Parlamentarismus, 5. erweiterte Auflage, Königstein 1980, S. 410 - 424, S. 410, unterscheidet zwischen klassisch parlamentarischer Opposition, Opposition aus Prinzip und einem Abklingen der Opposition als Folge politischer Kartellabsprachen. Heinrich Oberreuter (Hrsg.), Parlamentarische Opposition. Ein internationaler Vergleich, Hamburg 1975, S. 20, nimmt eine Differenzierung nach issue-orientierter Ad-Hoc-Opposition, kooperativer Opposition und kompetitiver Opposition vor. Winfried Steffani, Opposition, in: Kurt Sontheimer / Hans H. Röhring (Hrsg.), Handbuch des politischen Systems der Bundesrepublik Deutschland, 2. Auflage, München 1987, S. 427 - 433, S. 428, trennt zwischen den drei gegensätzlichen Begriffspaaren loyaler / fundamentaler Opposition; parlamentarischer / vor- beziehungsweise außerparlamentarischer Opposition ohne einen ausdrücklichen Wählerauftrag und systematischer/situationsorientierter Opposition, wobei letztere Oberreuters Ad-Hoc-Opposition vergleichbar ist.

14 Eine detaillierte Übersicht der unterschiedlichen Ansätze findet sich bei Ludger Helms, Wettbewerb und Kooperation. Zum Verhältnis von Regierungsmehrheit und Opposition im Parlamentarischen Gesetzgebungsverfahren in der Bundesrepublik Deutschland, Großbritannien und Österreich, Opladen 1997, S. 45 f.

15 So spricht Klaus von Beyme, Der Gesetzgeber. Der Bundestag als Entscheidungszentrum, Opladen 1997, S. 264, explizit von „parlamentarische[r] Mitregierung der Opposition“. Martin Sebaldt, Die Thematisierungsfunktion der Opposition. Die parlamentarische Minderheit des Deutschen Bundestags als innovative Kraft im politischen System der Bundesrepublik Deutschland, Frankfurt am Main 1992, schreibt der Opposition eine wichtige Steuerungsleistung im Vorfeld von 
ten feststellbar, die jeweils (wie zum Beispiel das britische Unterhaus) von nur einer Partei dominiert werden. ${ }^{16}$ Es scheint demnach plausibel, auch für die Opposition im Maximilianeum ein kooperatives Verhalten gegenüber der absoluten CSU-Mehrheit zu vermuten. Aber auch die mittlerweile seit über 40 Jahren währende Hegemonialstellung der Christsozialen spricht für die Kooperationshypothese: Angesichts dauerhaft ernüchternder Wahlergebnisse besteht für die Oppositionsparteien nur geringe Hoffnung darauf, die Regierungspartei abzulösen. ${ }^{17}$ Die „Alternativfunktion“ als eine der klassischen Aufgaben der Opposition entfällt damit weitgehend beziehungsweise bleibt vordergründig wirkungslos. Vielmehr scheint bei den gegebenen eindeutigen Mehrheitsverhältnissen den Oppositionsfraktionen in Bayern eine Mitwirkung an der Landespolitik ausschließlich durch Kooperation möglich. Damit stellt sich die Frage, ob sich die These vom Mitwirken der Opposition an der Gesetzgebung auch für den Bayerischen Landtag aufrechterhalten lässt und, falls ja, wie die Oppositionsparteien die Entscheidungen der Mehrheit beeinflussen.

„Politische Einflussnahme“ versucht die einschlägige Forschung in der Regel in ihren verschiedenen formalen und informalen Facetten durch andere Begriffe wie zum Beispiel „Initiativ- oder Thematisierungsfunktion“, „Agendasetting“ oder „Mitsteuerung“ zu umreißen. ${ }^{18}$ Das Herausgreifen dieser sehr unterschiedlichen Elemente deutet auf die Schwierigkeiten hin, ein gemeinsames definitorisches Dach für die Vielfalt politischer Beeinflussung zu finden. Hinter der begrifflichen Abgrenzungsproblematik verbirgt sich die forschungspraktische Schwierigkeit, die Mechanismen politischen Agierens und Gestaltens unter komplexen Bedingungskonstellationen methodisch zu erfassen, denn gerade im Bereich des Politischen lassen sich inhaltliche Erfolge oft nur schwer messen: So liegt dem Handeln von Politikern zwar in der Regel ein Leitfaden in Form eines Partei-, Wahl- oder Regierungs-

Entscheidungsprozessen durch die Thematisierung von Themen zu. Die Ansicht, gerade in der Ausschussarbeit "gelingt es häufig, noch Anträge der Opposition aufzunehmen“ (Manfred E. Berger, Renate Schmidt. „Was ich will“. Im Gespräch mit Manfred E. Berger, Düsseldorf 1994), wird von langjährigen Mitgliedern des Deutschen Bundestages immer wieder betont.

16 Vgl. Ludger Helms, a.a.O. (Fn. 14), S. 200, der allerdings mit einer sehr geringen Fallzahl operiert.

17 Als symptomatisch für die geringen Chancen der Opposition kann der Landtagswahlkampf 2003 gesehen werden, bei dem die Bayern-SPD unter dem Motto „Macht braucht Kontrolle“ auf ihren Wahlplakaten deutlich signalisierte, dass es ihr nicht darum ging, die CSU-Regierung abzulösen, als vielmehr eine Zweidrittelmehrheit der Christlichsozialen zu verhindern.

18 Allerdings können auch die von den jeweiligen Autoren geprägten unterschiedlichen Begriffsdefinitionen die Spannweite, innerhalb derer politische Einflussnahme stattfindet, nur bedingt umreißen. So erkennt Martin Sebaldt, a.a.O. (Fn. 15), S. 18, die Einflussnahme der Opposition auf die Politik der parlamentarischen Mehrheit in Form einer Initiativ- oder Thematisierungsfunktion, ohne diese allerdings näher zu definieren. Manfred Schwarzmeier, Parlamentarische Mitsteuerung. Strukturen und Prozesse informalen Einflusses im Deutschen Bundestag, Wiesbaden 2001, S. 50 f., der auf den von Sebaldt geprägten Begriff der politischen Steuerung als „systematische und geplante Lenkung des Prozesses der Vorbereitung, des Treffens und des Implementierens allgemein verbindlicher Entscheidungen " (Martin Sebaldt, a.a.O., S. 39 f.) rekurriert, sieht „Informales Mitsteuerungshandeln (...) charakterisiert durch eine unübersehbare Vielfalt“. Er definiert den Begriff der informalen Mitsteuerung als „diejenige parlamentarische Mitsteuerung, die durch Strukturen, Instrumente und Prozesse, die nicht durch formalrechtliche Vorgaben reguliert sind, ausgeübt wird“ (S. 50 f.). Auch Heinrich Oberreuter, Parlamentarische Opposition in der Bundesrepublik Deutschland, in: Walter Euchner (Hrsg.), Politische Opposition in Deutschland und im internationalen Vergleich, Göttingen 1993, S. 60 - 75, S. 74, der explizit die Frage nach dem „Einfluss“ der Opposition stellt, nimmt keine definitorische Abgrenzung des Begriffs vor. 
programms zu Grunde. Jedoch handelt es sich dabei zumeist um stark zugespitzte Absichtserklärungen oder vage Fernziele. Die in Wahlprogrammen plakativ getroffenen Versprechungen sind teilweise durch die damit intendierte Symbolkraft schlichtweg überhöht, oder es wird mit Zeitgeistbegriffen operiert, ohne diese näher zu definieren. ${ }^{19}$ Aber auch vollmundig geäußerte Regierungsziele lassen sich oft nicht realisieren. Rechtliche Vorgaben (zum Beispiel in Form bereits bestehender Rahmengesetze oder übergeordneter Verträge) und / oder Haushaltszwänge erschweren ihre sachpolitische Umsetzung. Zudem zwingen die Rücksichtnahme auf den Koalitionspartner, gegenläufige Mehrheiten im Bundesrat oder aber das Bestreben von Koordinierungsgremien der Länder, eine gemeinsame Position zu erlangen, zu Kompromissen. ${ }^{20}$ Dies schafft der Opposition im Gegenzug Einflussmöglichkeiten. Zuletzt kann die ex-post Betrachtung schwieriger Handlungsspielräume durch die mit Regierungs- und Oppositionsarbeit Befassten mitunter zu einer Um- oder Neudefinition eingangs geäußerter Zielvorstellungen und damit zu einer gänzlich neuen Bewertung der eigenen Einflussmöglichkeiten führen.

\section{Methode}

Angesichts dieses komplexen Wechselspiels unterschiedlicher Akteure, Interessen und Selbstdarstellungsweisen ist die Bewertung, ob ein geplantes Ziel durch die Politik erfolgreich umgesetzt wurde und welche Einflüsse in welchem Maße zu welchem Zeitpunkt Positionsveränderungen nötig machten, schwierig. Eine Einordnung des Grades politischer Einflussnahme in Form einer Skalierung oder der klaren Herausarbeitung spezieller Gesetzespassagen, die eindeutig auf einen bestimmten Urheber zurückzuführen sind, ist nur in seltenen Fällen möglich. Wie einschlägige Untersuchungen zum Bundestag zeigen, lassen sich das komplexe Zusammenspiel unterschiedlicher Bedingungskonstellationen und politischer Handlungsstrategien am ehesten durch einen multidimensionalen Ansatz und die Kombination unterschiedlicher qualitativer und quantitativer Untersuchungsmethoden herausarbeiten. ${ }^{21}$

Für eine erste Strukturierung des Materials wird im Folgenden daher zwischen direkter und indirekter Einflussnahme unterschieden. Direkte Einflussnahme bezieht sich auf Auseinandersetzungen zwischen den Fraktionen. Sie findet ihren Niederschlag beispielhaft in Form von Anträgen oder Plenarprotokollen. Die indirekte Einflussnahme steht dagegen für die Bedeutung, die Verbänden, Interessengruppen und / oder der Öffentlichkeit bei den Konflikten zwischen Opposition und Mehrheit zukommt. Auch wenn sich im politischen

19 Vgl. Ulrich von Alemann, Parteien und Medien, in: Oscar W. Gabriel / Oskar Niedermayer I Richard Stöss (Hrsg.), Parteiendemokratie in Deutschland, Bonn 2001, S. 467 - 483, S. 473 mit Verweis auf Otfried Jarren / Thorsten Grote / Christoph Rybarczyk, Medien und Politik - eine Problemskizze, in: Wolfgang Donsbach u.a. (Hrsg.), Beziehungsspiele - Medien und Politik in der öffentlichen Diskussion, Gütersloh 1993, S. 9 - 44, S. 19.

20 Vgl. zum Beispiel Manfred G. Schmidts These, Deutschland sei ein "Grand Coalition State“ (Manfred G. Schmidt, Germany. The Grand Coalition State, in: Joseph M. Colomer (Hrsg.), Political Institutions in Europe, London / New York 1996, S. 62 - 98) sowie Fritz Scharpfs Theorie der Politikverflechtung (Fritz Scharpf, Die Politikverflechtungs-Falle. Europäische Integration und deutscher Föderalismus im Vergleich, in: PVS, 26. Jg. (1985), S. 323 - 356).

21 Vgl. zum Beispiel Martin Sebaldt, a.a.O. (Fn. 15). 
Prozess beide Ebenen der Einflussnahmen vermischen, spannen sie einen Maßstab auf, an dem sich die Praxis messen lässt.

Obgleich es naheliegt, von der Existenz parteipolitisch unterschiedlicher Oppositionskulturen auszugehen, wurde dieser Aspekt in der politikwissenschaftlichen Forschung bislang vernachlässigt. Im Folgenden wird daher auch analysiert, ob die sozialdemokratische und die bündnisgrüne Fraktion im Maximilianeum eher eine konfliktorientierte Strategie in Form von heftigen Plenardebatten, der (Vor-)Verurteilung konkurrierender Entwürfe in Pressemeldungen und dem Beharren auf eigenen Alternativvorschlägen verfolgten oder in einer kooperativen Strategie der politischen Einflussnahme versuchten, Änderungen an den Entwürfen der Mehrheitsfraktion vor allem durch sachliche Überzeugungsarbeit in den Ausschussberatungen und die Vorlage konsensfähiger Kompromissvorschläge zu erreichen.

Als Untersuchungszeitraum wurde mit der 13. Legislaturperiode (1994 bis 1998) ein Abschnitt bayerischer Landtagsgeschichte gewählt, der lange genug zurück liegt, um eine gewisse Abgeschlossenheit der aus dem Landtagsarchiv und den Beständen der Verbände verfügbaren Akten zu gewährleisten ${ }^{22}$, und es zugleich erlaubt, die Interaktion von Regierungsmehrheit und Oppositionsparteien in den Kontext bundesdeutscher Politik ab Mitte der 1990er Jahre einzuordnen. Die Untersuchung beschränkte sich dabei auf die während der 13. Legislaturperiode im Landtag vertretenen Fraktionen von CSU, SPD und Bündnis 90/Die Grünen. ${ }^{23}$

Neben einer quantitativen Auswertung aller im Verlauf der 13. Legislaturperiode verabschiedeten Initiativen wurde der Verlauf mehrerer ausgewählter Gesetzgebungsverfahren detailliert nachgezeichnet und analysiert. Im Unterschied zu einigen Studien, die in den vergangenen Jahren oppositionelle Einflussmöglichkeiten im Bundestag untersucht haben ${ }^{24}$, wurde keine Zufallsstichprobe gezogen, sondern auf die theoriegeleitete Einhaltung bedeutsam erscheinender Parameter geachtet: Da im Zentrum der Untersuchung die Einflussnahme der Opposition auf das Regierungshandeln steht, wurden nur solche Verfahren ausgewählt, bei denen das politische Interesse durch eigene Gesetzesvorlagen sowohl der Regierungsmehrheit als auch der Opposition zum Ausdruck gebracht worden war und an deren Ende ein (zumeist auf einem Entwurf von Staatsregierung oder CSU-Fraktion basierendes) verabschiedetes Gesetz stand. ${ }^{25}$ Anhand der Auswahlkriterien kristallisierten sich

22 Demgegenüber blieb für die in Ministerien entstandenen Archivalien die 30-jährige Sperrfrist uneingeschränkt bestehen. Auch internes Material der einzelnen Fraktionen war leider nicht zugänglich.

23 Obgleich die nach der deutschen Einheit entstandene Vereinigung der Grünen mit der Bürgerrechtsbewegung Bündnis 90 den Parteinamen „Bündnis 90/Die Grünen“ trägt, bezeichnen sich die bündnisgrünen Abgeordneten im Bayerischen Landtag weiterhin als „Landtagsgrüne“ oder schlicht „Grüne“. Aus diesem Grund werden die unterschiedlichen Parteibezeichnungen synonym verwendet. In Fußnoten wird der Langtitel „Bündnis 90/Die Grünen“ mit der Kurzform „B90/Grüne“ abgekürzt.

24 So zum Beispiel Martin Sebaldt, a.a.O. (Fn. 15).

25 Aus systematischen Überlegungen ausgeschlossen blieben Verfahren, die sich mit der Arbeitssituation der Abgeordneten selbst beschäftigen, da anzunehmen ist, dass hier auf Grund des gemeinsamen Interesses aller Parlamentarier weitgehend im Konsens entschieden wurde. Ebenfalls nicht in die Analyse einbezogen wurden die Vorlagen, die im Zuge der im Februar 1998 vorgenommenen Verfassungsänderung von allen Fraktionen sehr zahlreich eingebracht wurden. Diese müssen gemäß Art. 75 Bayerische Verfassung mit einer Zweidrittelmehrheit im Landtag beschlossen und durch einen Volksentscheid legitimiert werden. 
aus den 181 Gesetzesinitiativen, über die der Landtag im Verlauf der 13. Legislaturperiode beraten hat, neun Themenkomplexe heraus, zu denen von den einzelnen Fraktionen beziehungsweise der Staatsregierung insgesamt 21 Gesetzesinitiativen eingereicht worden waren. Es wurden neben den Parlamentsmaterialien unter anderem Pressemeldungen, Medienberichte sowie die Stellungnahmen von Verbänden herangezogen. Zentrale Grundlage der Analyse waren darüber hinaus qualitative Interviews, bei denen insgesamt 21 Abgeordnete und Fraktionsmitarbeiter unter anderem zum Selbstverständnis ihrer Fraktion, ihrer Arbeitsweise und ihrer Bewertung der Oppositionspolitik befragt wurden. ${ }^{26}$

\section{Parameter oppositioneller Einflussnahme im Bayerischen Landtag}

Anhand der Aussagen von Abgeordneten sowie durch die quantitative und qualitative Auswertung von Parlamentspapieren konnten verschiedene Ebenen und Strategien oppositioneller Einflussnahme hinsichtlich der einzelnen Gesetzesinitiativen herausgearbeitet werden. Sie zeichnen sich durch ihre jeweils spezifische Kombination unterschiedlicher Orte und Modi der Beeinflussung aus. Während die machtpolitische Strategie ihren Akzent auf Konfrontation im Plenum des Landtags und Mobilisierung der Öffentlichkeit im vor- oder außerparlamentarischen Raum setzt, beschränkt sich die sachpolitische Strategie weitgehend auf den Landtag als Ort interfraktioneller Einflussnahme. Sie setzt dort verstärkt auf Kooperation in den Fachausschüssen und Einflussnahme im nicht-öffentlichen Bereich abseits der Gremien (vgl. Tabelle 1).

\begin{tabular}{|l|l|l|}
\hline Tabelle 1: Strategien oppositioneller Einflussnahme im Bayerischen Landtag \\
\hline & Modus der Auseinandersetzung & \multicolumn{1}{|l|}{ Ort der Auseinandersetzung } \\
\hline Sachpolitik & Kooperation & $\begin{array}{l}\text { Ausschüsse des Landtags, } \\
\text { informelle Kontakte abseits } \\
\text { der Gremien }\end{array}$ \\
\hline Machtpolitik & Konfrontation & $\begin{array}{l}\text { Plenum des Landtags, (Medien-) } \\
\text { Öffentlichkeit im vor- und } \\
\text { außerparlamentarischen Raum }\end{array}$ \\
\hline Quelle: Eigene Zusammenstellung. & \multicolumn{2}{|l}{} \\
\hline
\end{tabular}

Beide Strategien konnten gleichermaßen zu einer Positionsveränderung bei der CSU führen. Allerdings beschränkte sich der oppositionelle Erfolg in der Regel auf kleinere sachliche Änderungen in den Initiativen der Parlamentsmehrheit. Eine stärkere programmatische Einflussnahme auf die Politik der CSU-Mehrheit blieb SPD und Bündnis 90/Die Grünen im Bayerischen Landtag verwehrt.

26 Interviewleitfaden und Interviewcodes sind zu finden in: Katrin Steinack, a.a.O. (Fn. 11), S. 373 -376 . 


\subsection{Orte und Instrumente oppositioneller Einflussnahme}

Die Analyse der neun Gesetzgebungsprozesse zeigt, dass die Opposition sehr unterschiedliche Instrumente der Auseinandersetzung wählte. Sie lassen sich danach einordnen, ob sie eher auf der sach- oder machtpolitischen Ebene liegen und ob sie mehr dem öffentlichen oder nicht-öffentlichen Raum zuzurechnen sind (vgl. Tabelle 2). Die Instrumente machtpolitischer Einflussnahme sind ausschließlich im öffentlichen Raum, und dort außerhalb des Plenums, angesiedelt. Der Schwerpunkt sachpolitischen Einflusses liegt dagegen im nicht-öffentlichen Raum und in den (im Gegensatz zum Bundestag öffentlichen) Fachausschüssen. Das Fehlen von machtpolitischen Instrumenten im nicht-öffentlichen Raum lässt sich mit den Mehrheitsverhältnissen im Landtag und parlamentarischen Regelungen einerseits sowie der grundsätzlichen Rolle der Opposition andererseits erklären: Da der Bayerische Landtag bei Ausschusssitzungen die Öffentlichkeit zulässt, ist der nicht-öffentliche Raum, in dem die Abgeordneten agieren, kleiner als in anderen Parlamenten. Weil die Opposition einer Einparteiregierung gegenübersteht, schmälern sich ihre Möglichkeiten, diese - wie dies unter Umständen bei einer Koalitionsregierung der Fall wäre - über manche Themen zu spalten. Letztendlich bedarf das genuine Ziel jeder Opposition, die Macht zu erlangen, eines entsprechenden Wählervotums, dass am ehesten erreicht werden kann, wenn Defizite und Missstände der Regierung(spartei) so weit wie möglich öffentlich gemacht werden.

\begin{tabular}{|c|c|c|}
\hline & sachpolitische Ebene & machtpolitische Ebene \\
\hline $\begin{array}{l}\text { nicht- } \\
\text { öffentlicher } \\
\text { Raum }\end{array}$ & $\begin{array}{l}\text { - fraktionsübergreifende Gespräche von } \\
\text { Sachpolitikern abseits von Ausschuss- } \\
\text { und Plenarsitzungen } \\
\text { - Lobbyarbeit von Interessenvertretern } \\
\text { zur Überzeugung der parlamentari- } \\
\text { schen Mehrheit } \\
\text { - Diskussion von Anträgen im } \\
\text { Ausschus** }\end{array}$ & \\
\hline $\begin{array}{l}\text { öffentlicher } \\
\text { Raum }\end{array}$ & $\begin{array}{l}\text { - Änderungsanträge zu Vorlagen der } \\
\text { parlamentarischen Mehrheit } \\
\text { - Anhörung von Experten und } \\
\text { Interessenvertretern } \\
\text { - Diskussion im Plenum }\end{array}$ & $\begin{array}{l}\text { - Lancierung von Medienberichten durch } \\
\text { Pressemeldungen der Fraktionen zur } \\
\text { Information der Öffentlichkeit } \\
\text { - Unterstützung der parteipolitischen } \\
\text { Positionen in den Medien durch } \\
\text { Interessenvertreter } \\
\text { - Initiative von Petitionen und/oder } \\
\text { Volksbegehren (unter Umständen } \\
\text { gemeinsam mit Interessenvertretern) } \\
\text { - Initiierung von Aktuellen Stunden zur } \\
\text { Konfrontation der Regierung mit } \\
\text { oppositionellen Konzepten }\end{array}$ \\
\hline \multicolumn{3}{|c|}{$\begin{array}{l}\text { * Die Ausschusssitzungen sind zwar formal öffentlich, faktisch ist die Öffentlichkeit (zum Beispiel } \\
\text { Besuchergruppen oder Journalisten) jedoch oft nicht anwesend. } \\
\text { Quelle: Eigene Zusammenstellung. }\end{array}$} \\
\hline
\end{tabular}


Auch wenn die beiden Ebenen der Einflussnahme von den befragten Abgeordneten nicht explizit so benannt wurden, ergab sich aus ihren Aussagen und der Auswertung des empirischen Materials, dass an den verschiedenen Orten der politischen Auseinandersetzung unterschiedliche Strategien zur Anwendung kommen.

Die Interviewpartner sahen das Landtagsplenum vorrangig als Ort der Konfrontation, in dem die Abgeordneten unter parteipolitischen Vorzeichen aufeinander treffen. ${ }^{27}$ Eine Diskussion politischer Inhalte fand dort in der Regel in Form der zweiten (sowie seltener der dritten) Lesung von Gesetzesinitiativen statt. Darüber hinaus nutzte die Opposition die Möglichkeit, die Regierungspolitik in Aktuellen Stunden kritisch zu hinterfragen. Dabei zeigte die quantitative Analyse der Plenardebatten in der zweiten Jahreshälfte 1996, dass das Plenum von Stellungnahmen der Staatsregierung dominiert wurde. ${ }^{28}$ Zugleich untermauert die qualitative Analyse einzelner Gesetzgebungsprozesse den Charakter des Plenums als Ort, an dem vor allem eigene Positionen dargestellt wurden. Eine sachliche Auseinandersetzung mit den Vorschlägen des politischen Gegners erfolgte dort kaum. ${ }^{29}$

Dagegen waren die Ausschusssitzungen zumindest quantitativ stark durch die Einflussversuche von SPD und Bündnis 90/Die Grünen geprägt. Die Regierung dominierte zwar den Bereich der Gesetzesvorlagen; abgesehen davon waren jedoch über 70 Prozent der im Verlauf der Legislaturperiode insgesamt behandelten Initiativen von der Opposition ausgegangen. ${ }^{30}$ Zudem betonten die Abgeordneten, dass oppositionelle Einflussnahme am ehesten in den Ausschüssen möglich sei. ${ }^{31}$ Dabei ist jedoch zu bedenken, dass von diesen nur Empfehlungen in Bezug auf Zustimmung oder Ablehnung einer Vorlage getroffen werden konnten. Die Entscheidung darüber, ob eine Initiative letztlich angenommen wurde, lag beim Plenum, wobei auch eine Summe positiver Ausschussempfehlungen nicht automatisch die Zustimmung der parlamentarischen Mehrheit nach sich zog.

Dritte wesentliche Komponente oppositioneller Einflussnahme war der Appell an die sowie die Einbindung der Öffentlichkeit. Die informelle Beeinflussung der Regierungspolitik im vor- und außerparlamentarischen Raum wurde in den Abgeordneteninterviews fraktionsübergreifend als das wichtigste Instrument oppositioneller Arbeit bewertet. ${ }^{32}$ Während im Vorfeld von Gesetzesinitiativen vor allem Verbandskontakte sehr bedeutend sind, versuchen die Abgeordneten nach formalem Beginn der Gesetzgebungsverfahren, die Wähler durch Pressemeldungen und Medienberichte zu informieren. Dies kann in Ausnahmefällen derart instrumentalisiert werden, dass sich Teile der Bevölkerung mit Petitionen oder in Form eines Volksbegehrens an den Gesetzgeber wenden und damit die Opposition unterstützen.

27 Vgl. Interview 01-290101 B90/Grüne, Z. 80 ff.

28 Vgl. Katrin Steinack, a.a.O. (Fn. 11), S. $142 \mathrm{ff}$.

29 Am deutlichsten wurde dies bei den Diskussionen der Parlamentarier über die Einführung des Kommunalwahlrechts für EU-Ausländer, bei dem die CSU-Vertreter vor allem die potentielle Benachteiligung deutscher Wähler bemängelten. Demgegenüber stellten die bündnisgrünen Abgeordneten die ihres Erachtens diskriminierende Ausgrenzung von Ausländern durch die Regierungsmehrheit an den Pranger. Die konkrete Umsetzung der EU-Richtlinie war hingegen kaum Gegenstand der Diskussion. Vgl. Katrin Steinack, a.a.O. (Fn. 11), S. 203 ff.

30 Vgl. Katrin Steinack, a.a.O. (Fn. 11), S. 133.

31 Vgl. Interview 08-150201 SPD, Z. $93 \mathrm{ff}$.

32 Vgl. zum Beispiel die Interviews 05-050201 SPD, Z. 354 ff.; 20-140301 CSU, Z. 508 ff.; 01290101 B90/Grüne, Z. 11 ff.; 17-190301B90/Grüne, Z. 200 ff. 


\subsection{Oppositionelle Strategien zur Durchsetzung politischer Interessen}

Die stark öffentlichkeitsorientierte Diskussion politischer Themen im Plenum und im vorund außerparlamentarischen Rahmen sowie die sachliche Auseinandersetzung mit Kollegen im Ausschuss oder sogar schon im Vorfeld von Ausschusssitzungen markieren zwei gegensätzliche Pole oppositioneller Einflussstrategien. Beide Wege wurden gleichermaßen von den Abgeordneten der Opposition genutzt. Von den neun untersuchten Verfahren konnten je vier eindeutig als sachpolitisch beziehungsweise machtpolitisch dominierte Verfahren identifiziert werden. Die Auseinandersetzungen um das Naturschutzgesetz fanden dagegen fast gleichermaßen auf sach- wie machtpolitischer Ebene statt. ${ }^{33}$ Dabei wurde die Entscheidung der Politiker für eine bestimmte Strategie oppositioneller Einflussnahme wohl nur in wenigen Fällen bewusst getroffen. Vielmehr ziehen bestimmte Themenfelder oder Interessenkonstellationen zwangsläufig bestimmte politische Handlungsmuster nach sich.

Die Abgeordneten wählten die sachpolitische Strategie, wenn ein Thema vergleichsweise komplex war und damit nicht der Anforderung einer leichten medialen Vermittelbarkeit entsprach. Dies war zum Beispiel bei den Gesetzen zur Vermeidung von Fehlsubventionierung im sozialen Wohnungsbau oder zur Änderung der Immissionsschutzbestimmungen der Fall. Aber auch bei relativ einfach darzustellenden Belangen, beispielsweise der Verankerung des Breitensports als Soll-Aufgabe, fand eine stillschweigende Kooperation von Regierungsmehrheit und Opposition statt. Wesentliche Grundlage der parteiübergreifenden Zusammenarbeit war, dass es sich bei dem Gesetzgebungsgegenstand um einen Sachverhalt allgemeinen Interesses handelte, der von keiner der Parteien programmatisch besetzt war und sich keiner parteispezifischen Wählerklientel eindeutig zurechnen ließ.

Im Gegensatz dazu wurde die machtpolitische Strategie bei Themen angewandt, die hohe gesellschaftliche Relevanz besaßen und einen engen Bezug zur parteipolitischen Programmatik aufwiesen. Die untersuchten Verfahren, die der machtpolitischen Ebene zugeordnet werden konnten, befassten sich fast sämtlich mit Themen, die wie die Frage der Gleichstellung der Geschlechter, die Integration von Ausländern oder die Nutzung der Gentechnik mit weltanschaulichen Grundpositionen der Parteien verknüpft waren. Wichtig war zudem, dass die jeweilige Materie der Bevölkerung in wenigen Stichworten vermittelt beziehungsweise mit klaren Aussagen besetzt werden konnte.

\subsection{Bedingungsfaktoren des oppositionellen Erfolgs}

Den Erfolg der oppositionellen Einflussversuche in der Gesetzgebung exakt zu beziffern ist schwierig. Die Interviewpartner aus SPD und Bündnis 90/Die Grünen werteten bereits die Tatsache, dass die CSU-Mehrheit in Folge eines Gesetzentwurfs der Opposition dazu bewegt werden konnte, zu einem Thema einen eigenen Entwurf vorzulegen, als Erfolg. ${ }^{34} \mathrm{Al}-$ lerdings wurden nur in wenigen Fällen Passagen und Forderungen aus den Entwürfen der Opposition in die letztlich verabschiedete Vorlage der parlamentarischen Mehrheit übernommen. Für die vorliegende Untersuchung waren von vornherein nur solche Gesetzesinitiativen ausgewählt worden, bei denen sowohl von der Regierungsmehrheit als auch von 
mindestens einer der Oppositionsfraktionen ein Entwurf eingebracht worden war. Damit konzentriert sich die Bewertung des Erfolges auf die Frage, inwieweit die Opposition konkrete inhaltliche Forderungen durchsetzen konnte beziehungsweise inwieweit sich oppositionelle Vorschläge im verabschiedeten Gesetz wiederfinden.

Nachfolgend wird nach Erfolg, Teilerfolg und Misserfolg differenziert (vgl. Tabelle 3 mit Beispielen): Erfolg hatte die Opposition dann, wenn es ihr gelang, das verabschiedete Gesetz maßgeblich zu prägen. Dies drückt sich darin aus, dass weite Passagen der oppositionellen Vorlage mit dem verabschiedeten Gesetz übereinstimmen. Einen Teilerfolg konnte die Opposition erzielen, wenn sie einzelne Forderungen, die ursprünglich von der Regierungsmehrheit zurückgewiesen worden waren, in deren Vorlage festschreiben konnte. Einen Misserfolg verzeichnete die Opposition, wenn sie trotz umfangreicher Bemühungen keine einzige ihrer Forderungen durchsetzen konnte und die CSU-Vorlage gänzlich unverändert verabschiedet wurde.

Die kooperative Strategie war für die Opposition von Erfolg gekrönt, wenn auch die CSU die Befassung mit der Problematik als wichtig erachtete und entsprechend initiativ wurde. Grundlage der Kooperation war damit die gemeinsame Überzeugung, dass es nötig sei, einen Sachverhalt schnell zu klären (beispielsweise beim Immissionsschutz oder bei der Frage des Breitensports). Dass bei den untersuchten kooperativen Verfahren nur eine geringe oder gar keine Pressearbeit feststellbar war, weist darauf hin, wie wichtig die Vermeidung von Öffentlichkeit für die parteiübergreifende Kooperation ist. So stellte eine bündnisgrüne Parlamentarierin gerade die Gewährleistung von Nichtöffentlichkeit als wichtigsten Garanten für eine erfolgreiche Zusammenarbeit mit der CSU heraus. ${ }^{35}$

\begin{tabular}{|l|l|l|l|}
\hline \multicolumn{4}{|c|}{ Tabelle 3: Erfolg der kooperativen Oppositionsstrategie am Beispiel der } \\
im Bayerischen Landtag 1994 bis 1998
\end{tabular}

$\mathrm{Zu}$ bedenken ist bei den oppositionellen Erfolgen im kooperativen Bereich allerdings, dass diese erzielt wurden, wenn die Materie (wie die Aufwertung des Breitensports) parteiübergreifend unstrittig und programmatisch unverdächtig war. Nur in diesen Fällen konnte die Zustimmung der Mehrheitsfraktion erlangt werden. Der Sieg der Sozialdemokraten, deren ursprünglicher Antrag zur Änderung der Gemeindeordnung von der CSU-Fraktion fast wortwörtlich übernommen worden war, wiegt damit aus parteipolitischer Sicht gering. Die Diskussionen über die Veräußerung staatlicher Grundstücke, bei denen die unterschied- 
lichen Sozialkonzepte der beiden Volksparteien aufeinander prallten, zeigt, wie schnell die Einflussmöglichkeiten der SPD endeten, sobald auch nur ansatzweise programmatisch relevante Themen angeschnitten wurden.

Während beim kooperativen Zusammenspiel der Landtagsfraktionen für die Opposition Einflussnahme nur dann möglich war, wenn keine Themen von parteipolitischem Gewicht tangiert wurden, setzte die konfrontative Strategie darauf, explizit unterschiedliche politische Ansätze der Parteien zu einem Thema zu kontrastieren und die Bevölkerung von der besseren Eignung des oppositionellen Konzepts zu überzeugen (vgl. Tabelle 4 mit Beispielen). Wie bei der kooperativen Strategie waren auch hier unterschiedliche Stufen oppositioneller Einflussnahme feststellbar: Die Strategie der öffentlichkeitswirksamen Konfrontation war für die Opposition erfolgreich, wenn es ihr gelang, Betroffenheit in der Bevölkerung herzustellen und somit Druck für die CSU entstand, die Angelegenheit in Form eines eigenen Gesetzentwurfs zu regeln. Die Betroffenheit wurde entweder durch einen persönlichen Bezug der Wähler zum Thema (wie beim Gentechnikgesetz) oder durch das Schüren von Emotionen (zum Beispiel beim Jagdgesetz) hergestellt. Dabei kam der Beteiligung der Presse für das Schaffen von Öffentlichkeit eine zentrale Rolle zu.

Wenn es darum ging, die CSU über das eigene Initiativwerden hinaus zur Übernahme oppositioneller Forderungen zu bewegen, konnte die Opposition lediglich Teilerfolge erzielen. So gelang es den Abgeordneten von Bündnis 90/Die Grünen beispielsweise im Jagdgesetz, den Radius, innerhalb dessen freilaufende Hunde nicht abgeschossen werden dürfen, zu vergrößern. In Bezug auf den Status der Gleichstellungsbeauftragten konnten beide Oppositionsfraktionen gemeinsam die Festschreibung der politischen Unabhängigkeit der Beauftragten erreichen.

Wichtig für den Positionswechsel der Mehrheitsfraktion waren in beiden Fällen die außerparlamentarischen Allianzen der Opposition. Sie konnte im Fall des Jagdgesetzes auf die

\begin{tabular}{|c|c|c|c|}
\hline \multirow[t]{2}{*}{ Tabelle 4: } & \multirow[b]{2}{*}{ Erfolg } & \multirow{3}{*}{$\begin{array}{l}\text { Teilerfolg } \\
\text { Kennzeichnung } \\
\text { gentechnisch nicht } \\
\text { veränderter Lebensmittel } \\
\text { aus Bayern } \\
\text { Gleichstellung von } \\
\text { Frauen und Männern } \\
\text { im Öffentlichen Dienst }\end{array}$} & ppositions-fraktionen \\
\hline & & & Misserfolg \\
\hline SPD & & & \\
\hline $\begin{array}{l}\text { Bündnis 90/Die } \\
\text { Grünen }\end{array}$ & & $\begin{array}{l}\text { Änderung des Bayeri- } \\
\text { schen Jagdgesetzes } \\
\text { Kennzeichnung } \\
\text { gentechnisch nicht } \\
\text { veränderter Lebensmittel } \\
\text { aus Bayern } \\
\text { Gleichstellung von } \\
\text { Frauen und Männern } \\
\text { im Öffentlichen Dienst }\end{array}$ & $\begin{array}{l}\text { Änderung des } \\
\text { Gemeinde- und } \\
\text { Landkreiswahlgesetzes }\end{array}$ \\
\hline
\end{tabular}


Unterstützung durch zahlreiche Petitionen von Tierschützern und bei der Gleichstellung auf die Zustimmung zahlreicher Verbände aus dem christlich-konservativen Milieu verweisen. An der Gesamtkonzeption der Gesetze änderten die von der Opposition erzielten Modifikationen jedoch nichts. Zudem zeigt das Beispiel des Kommunalwahlrechts für EUAusländer, bei dem die Opposition keine einzige ihrer Forderungen durchsetzen konnte, dass auch eine große Presseresonanz sowie die Unterstützung zahlreicher Verbände vergeblich waren, wenn damit nicht auch die Wählerklientel der CSU angesprochen wurde. Dies war bei der Einführung des Ausländerwahlrechts, für die sich vor allem SPD-Politiker und Ausländerbeauftragte ausgesprochen hatten, nicht der Fall. ${ }^{36}$

\section{Parteipolitische Unterschiede in der Arbeit der Opposition}

Besonderheiten im Auftreten der beiden Oppositionsfraktionen wurden erstens von den interviewten Abgeordneten benannt. Insbesondere die CSU-Vertreter empfanden ihre bündnisgrünen Kollegen als unerschrockener, engagierter und agiler als die der SPD. Vielfach vertraten sie die Einschätzung, es sei vor allem die bündnisgrüne Fraktion gewesen, die in der 13. Legislaturperiode Oppositionsarbeit geleistet und eine konfrontative Haltung zur Staatsregierung eingenommen habe. Die SPD-Mitglieder wurden als gehemmter und weniger dynamisch im Auftritt geschildert. Gleichzeitig wurde ihre größere Bereitschaft zur Kooperation hervorgehoben.

Zweitens zeigte die Auswertung der neun Gesetzgebungsverfahren, dass SPD und Bündnis 90/Die Grünen zur Umsetzung ihrer Ziele tatsächlich unterschiedlich agierten. Dies deutet darauf hin, dass grundsätzlich verschiedene Politische Kulturen im oppositionellen Verhalten der beiden Parteien bestehen, die auch über die 13. Legislaturperiode hinaus nachweisbar sind.

\subsection{Selbst- und Fremdwahrnehmung der Oppositionsfraktionen}

In den Beschreibungen der Interviewpartner gewannen die Vertreter von Bündnis 90/Die Grünen am meisten Kontur. Sie wurden im Vergleich zu den Parlamentariern von SPD und CSU als „sehr intensive Persönlichkeiten“37, im Auftreten individueller und im Gesamteindruck bunter empfunden. Einige Abgeordnete sahen dies als Besonderheit, die umgekehrt proportional zur Fraktionsgröße stehe. ${ }^{38}$ Die exponierte Stellung der grünen Abgeordneten in den Ausschüssen war ihrer Ansicht nach der wesentliche Grund dafür, dass diese in ihrem Auftreten als streitbarer und insgesamt profilierter empfunden wurden: Allein durch seine Wortmeldung - so der Erklärungsansatz einer Sozialdemokratin - rücke der jeweilige Ausschussvertreter bereits in eine herausgehobene Gegenposition zu den Sprechern der beiden anderen Fraktionen. ${ }^{39}$ Dass der großen Gruppe christlich-sozialer Abgeordneter im Ausschuss neben einigen Sozialdemokraten jeweils nur ein grüner Vertreter gegenübersaß, dem es oblag, die gesamte inhaltliche Palette der Ausschussarbeit abzudecken, nötigte vor allem CSU-Parlamentariern Respekt ab:

36 Vgl. Katrin Steinack, a.a.O. (Fn. 11), S. 203 ff.

37 Interview 05-050201 SPD, Z. $274 \mathrm{ff}$.

38 Vgl. Interview 01-290101 B90/Grüne, Z. 188 f.; Interview 09-150201 SPD, Z. 73 ff.

39 Vgl. Interview 11-190201 SPD, Z. $120 \mathrm{ff}$. 
„Die grünen Abgeordneten haben natürlich wahnsinnig zu ackern, können sich Ausfälle überhaupt nicht leisten, sind immer allein, auch in den Ausschüssen. Wenn spezielle Ausschussarbeit getan wird, picken [sie] sich dann natürlich speziell ihre Sachen raus, können so nur überleben, sonst gehen sie ja unter in der Fülle der einzelnen Punkte, die da sind. Also, die Grünen werden sicherlich als Opposition auch stärker wahrgenommen. Thematisch (...) und da bewundere ich manche schon, wie sie das dann schaffen. Da gehört ja schon einiges an Durchhaltevermögen dazu. Deswegen sind sie dann auch meines Erachtens viel bissiger, also auch zupackender." 40

Durch die Konzentration auf wenige Teilbereiche, in denen sie durch souveräne Argumentation überzeugten, wurde den grünen Abgeordneten von der Regierungsmehrheit ein deutlich eigenständigeres programmatisches Auftreten zugeschrieben als den sozialdemokratischen Kollegen. Sie wurden in Interviews mehrfach als „eigentliche Opposition“ hervorgehoben, der es bei Sachfragen gut gelungen sei, Alternativpositionen pointiert zu artikulieren und damit das parteipolitische Profil der gesamten Fraktion zu schärfen. ${ }^{41}$ Die Bürde, in einem Ausschuss allein für die Vertretung grüner Positionen verantwortlich zu sein, konnten sie somit in einen Vorteil verwandeln. Dabei ermöglichte gerade der Verzicht auf eine zeitraubende und teilweise auch Ideen verwässernde Abstimmung einzelner Abgeordneter untereinander im Arbeitskreis, wie dies für die beiden großen Fraktionen der Fall ist, den Landtagsgrünen eine agilere und frechere Oppositionspolitik. ${ }^{42}$

Ein wesentlicher Bonus der grünen Fraktion waren nach Ansicht von SPD-Abgeordneten zudem deren effizientere Kommunikationsstrukturen und bessere, teilweise auch kreativere, Kontakte zur Presse. ${ }^{43}$ Demgegenüber würde die SPD, so die Selbstwahrnehmung, - ungeachtet ihrer tatsächlichen politischen Ansätze und Ziele - in der veröffentlichten Meinung „immer eher als etwas langweilig“ 44 geschildert. Obwohl die Fraktion politische Positionen durch einen größeren Stab an Mitarbeitern erarbeiten und fachlich untermauern lassen kann und über eine umfangreiche Pressestelle verfügt, wurde ihre Außendarstellung von vielen Abgeordneten als mangelhaft empfunden, wie ein Sozialdemokrat beklagt: „Es ist einer der größten Trauerpunkte innerhalb der Fraktion, dass die Öffentlichkeitsarbeit zu schlecht ist. Wenn die Leute nur verstehen würden, was wir alles Gutes vorhaben, könnte es doch gar nicht sein, dass jemand eine andere Partei wählt als die SPD. “45 Wesentlicher Grund der mäßigen Resonanz, die seine Partei in der Öffentlichkeit erzielte, waren seiner Auffassung nach überkommene Kommunikationsstrukturen:

„[Die SPD hat], übrigens ähnlich wie andere Massenorganisationen, wie Kirchen und Gewerkschaften lange Zeit, (...) eine Kommunikationsstruktur erfolgreich gehabt, die sich in der heutigen Kommunikationsgesellschaft als relativ unerfolgreich herausstellt. (...) Die Grünen haben den Vorteil, überhaupt nie solche Kommunikationsstrukturen gehabt zu haben. Am deutlichsten sieht man das bei Greenpeace und Amnesty International und ähnlichen; die hatten von Anfang an ein Kommunikationsverhalten, das sehr viel stärker auf die heutige Medienkommunikationsstruktur angepasst war. Wenn ich nämlich darlegen will, dass die

40 Interview 04-010201 CSU, Z. $198 \mathrm{ff}$.

41 Vgl. Interview 04-010201 CSU, Z. 168 ff.; Interview 07-150201 CSU, Z. 218 ff.; Interview 13$120301 \mathrm{CSU}$, Z. $248 \mathrm{ff}$.

42 Vgl. Interview 07-150201 CSU, Z. 227 ff. und Interview 05-050201 SPD, Z. $264 \mathrm{ff}$.

43 Vgl. die Interviews 05-050201 SPD, Z. 393 ff. und 18-240401 SPD, Z. 202 ff.

44 Interview 18-240401 SPD, Z. $200 \mathrm{ff}$.

45 Interview 05-050201 SPD, Z. $385 \mathrm{ff}$. 
chemische Industrie eigentlich zu viel Abgase erzeugt, schreib ich nicht erst ein langes Papier und gib es sämtlichen Redakteuren und ich mach auch keinen Kongress, sondern ich häng mich an einen Schornstein hin und bekomme damit die Öffentlichkeit. (...) Gerade die bayerische SPD ist eigentlich in ihrem Kommunikationsverhalten noch immer geprägt von vor 50 Jahren oder so.“46

Auch als Individuen blieben die sozialdemokratischen Abgeordneten in der Darstellung der Interviewpartner blass und gewannen kaum Kontur. Begriffe wie „kreativ“, „individuell“ oder „unkonventionell“ wurden für sie nicht gebraucht. In den Schilderungen der Interviewten wirkte die Fraktion vielmehr wie eine vom Geist des Öffentlichen Dienstes geprägte, wenig dynamische Gruppe, in der sich bedingt durch eine teilweise Ermüdung der Parlamentarier, die bereits durch eine lange Parteikarriere verschlissen sind, nur ein kleiner Teil engagiert der Oppositionsarbeit widmete. Ein SPD-Abgeordneter mahnte diesbezüglich die seines Erachtens gegebene Überalterung seiner Fraktion sowie die durch die lange Oppositionszeit fehlende Motivation zur politischen und personellen Erneuerung an:

„Die SPD ist nicht attraktiv für dynamische oder jüngere Personen (...) Sie müssen sich vorstellen: wenn jemand mit 25 Lehrer wird und dann zur SPD geht, dann kann er entweder zum Ortsverein immer ganz brav und aktiv gehen; das wird aber auch mit der Zeit langweilig. Und für einen Lehrer ist es auch finanziell attraktiv, Landtagsabgeordneter zu werden, selbst wenn er nichts wird. Es ist aber dann auch wichtig, wenn er drin ist, wieder reinzukommen. Also muss er im Wesentlichen seinen Wahlkreis verteidigen und sehen, dass er innerhalb der Partei nicht abrutscht, was sowieso nicht vorkommt; wer mal drin ist, bleibt drin. Sie [die Abgeordneten] haben natürlich im Wesentlichen eine Grundeinstellung, die davon ausgeht, ja nichts zu ändern, denn sonst fällt man noch unter die Räder. Wenn wir da noch ein paar dynamische Junge reinlassen, was passiert denn da mit uns? “47

Nach außen hin versuchte die SPD-Fraktion nach Ansicht der Abgeordneten weit stärker als die Grünen, homogen zu wirken, ein Anspruch, der - auch dies anders als bei der Ökopartei - vor allem mit den Erwartungen der Wählerschaft begründet wurde. ${ }^{48} \mathrm{Da}$ die Sozialdemokraten in der 13. Legislaturperiode jeweils mit mehreren Abgeordneten in den Ausschüssen vertreten waren, konnte eine stärkere Arbeitsteilung bei gleichzeitig intensiver Bearbeitung der behandelten Themenfelder vorgenommen werden. Um trotz dieser Expertenbildung in den Ausschussberatungen gegenüber CSU und Grünen geschlossen auftreten zu können, war allerdings eine stärkere parteiinterne Abstimmung in Arbeitskreis- und Fraktionssitzungen nötig, bei der Extrem- und Außenseiterpositionen in der Regel zugunsten einer zumeist verbindlichen Mehrheitsmeinung geglättet wurden. ${ }^{49}$ Der Preis, den die Sozialdemokraten dafür bezahlten, war eine erschwerte Abgrenzung und Profilbildung gegenüber der CSU. In Sachfragen zeigten sie nach Ansicht der Abgeordneten von Grünen und CSU deutlich weniger Kontur als die kleinere Oppositionspartei. Zugleich zeichnete

46 Interview 05-050201 SPD, Z. $393 \mathrm{ff}$.

47 Interview 05-050201 SPD, Z. $500 \mathrm{ff}$.

48 Vgl. Interview 21-151001 SPD, Z. $239 \mathrm{ff}$.

49 Abstimmungen, in denen einzelne Abgeordnete ohne Rücksicht auf die Fraktionsmeinung frei entscheiden können, sind selten. Vgl. Interview 21-151001 SPD, Z. 315 sowie Interview 01290101 B90/Grüne, Z. 574, in dem darauf hingewiesen wurde, dass bei starken Differenzen in einer Fraktion über einen Aspekt auf dessen Thematisierung schlichtweg verzichtet werden würde. Die Entscheidung über das gemeinsame Abstimmungsverhalten in Ausschuss- und Plenarberatungen wird in der Regel während der wöchentlichen Fraktionssitzungen getroffen. 
sich die Fraktion durch eine größere Bereitschaft zur Kooperation mit der Regierungsmehrheit aus und wurde nach Aussagen von CSU-Vertretern von dieser häufiger in ihre politischen Entscheidungen eingebunden: Anders als mit den Grünen könne man mit den Sozialdemokraten nämlich, so die Ansicht einer CSU-Politikerin, „zu Rande kommen, und mit denen kommen Sie auch leichter zu Rande. Weil die, selbst wenn es da einige Extrempositionen gibt bei der SPD, angesichts der doch größeren Zahl der Abgeordneten in der Summe wieder ausgewogener sind“ 50 .

Neben dem programmatischen Verzicht auf extreme politische Forderungen scheinen für die größere Kooperations- und Kompromissbereitschaft der SPD-Fraktion zwei weitere Gründe ausschlaggebend: Zum einen trägt nach Ansicht eines CSU-Politikers der stärkere Praxisbezug sozialdemokratischer Parlamentarier durch die häufigere Übernahme kommunaler Mandate und Ämter, aber auch der Austausch mit Sozialdemokraten, die in anderen Bundesländern in der Regierungsverantwortung stehen, zu einer grundsätzlich pragmatischeren Haltung der Fraktion bei. So nehme die SPD-Fraktion trotz opponierender Haltung zur CSU „schon auch Rücksicht auf die praktische Durchsetzbarkeit“"51 einzelner Forderungen. Ein weiterer Grund für die größere Bereitschaft, sich auf die CSU hin zu bewegen, lag nach Ansicht eines SPD-Vertreters darin, dass die Abgeordneten, durch langjährige Opposition resigniert, eher kompromissbereit seien als die grüne Fraktion - gemäß der Devise: „Ich will einen Erfolg sehen und nicht immer nur Niederlagen. "52

\subsection{Strategische Präferenzen der Oppositionsfraktionen in der Gesetzgebung}

Die Aussagen der Interviewpartner zum unterschiedlichen Auftreten von Sozialdemokraten und Grünen korrespondieren mit dem Verhalten der beiden Fraktionen in den ausgewählten Gesetzgebungsprozessen, was als zweiter zentraler Indikator für die strategischen Präferenzen der Oppositionsparteien (vgl. Tabelle 5) gewichtet wurde: In drei der vier sachpolitischen Verfahren wurde ein SPD-Antrag, nur einmal ein Entwurf von Bündnis 90/Die Grünen verhandelt. Dies war zugleich der einzige untersuchte Fall, bei dem zuerst eine Regierungsvorlage und erst danach eine Initiative aus den Reihen der Opposition vorgelegt worden war. Das verzögerte Initiativwerden der Bündnisgrünen in diesem Zusammenhang ist mit ein Zeichen dafür, dass die Ökopartei nicht vorrangig auf sachpolitische Einflussnahme setzte.

Bei allen vier Gesetzgebungsprozessen mit einer stark machtpolitischen Konnotation hatte hingegen zuerst ein Entwurf der bündnisgrünen Fraktion vorgelegen, auf den dann eine Initiative der Regierungsmehrheit folgte. Die SPD war nur in einem machtpolitisch geprägten Verfahren, zur Gleichstellung der Geschlechter im Öffentlichen Dienst, mit einem eigenständigen Gesetzentwurf präsent. ${ }^{53}$

Bei der Änderung des Bayerischen Naturschutzgesetzes, das als Mischform zwischen Macht- und Sachpolitik eingeordnet worden ist, wurde die bündnisgrüne Gesetzesvorlage

50 Interview 10-160201 CSU, Z. $263 \mathrm{f}$.

51 Interview 04-012001 CSU, Z. $194 \mathrm{ff}$.

52 Interview 08-150201 SPD, Z. $187 \mathrm{ff}$.

53 In einem weiteren Verfahren zum Bereich Gentechnik hatte sich die SPD-Fraktion der Gesetzesinitiative der Bündnisgrünen angeschlossen, war aber nicht originär tätig geworden. 
zwar in Parteipublikationen diskutiert, in der breiteren Öffentlichkeit wurde die Gesetzesänderung allerdings kaum wahrgenommen.

Die Auswertung der Verfahren zeigt, dass Bündnis 90/Die Grünen beim Versuch, ihre Initiativen durchzusetzen, eine machtpolitisch orientierte Strategie der öffentlichkeitswirksamen Konfrontation bevorzugten. Im Gegensatz dazu agierten die Sozialdemokraten stärker sachpolitisch und setzten überwiegend auf interne Einflussnahme. Dabei suchten sie in der Regel die Kooperation im Ausschuss. Dies galt auch dann, wenn die SPD keine eigene Gesetzesinitiative vorgelegt hatte. So versuchten die Fraktionsmitglieder beim Naturschutzgesetz durch eine Fülle von Anträgen, den CSU-Entwurf sachlich abzuändern. Bei den stark emotionalisierten Debatten über das Jagdgesetz vermittelten die sozialdemokratischen Parlamentarier zwischen den gegensätzlichen Meinungen von Abgeordneten der CSU und Bündnis 90/Die Grünen. Ihr kooperatives Engagement bestätigt damit die Ergebnisse der quantitativen Analyse aller Initiativen der 13. Legislaturperiode, die eine deutlich höhere Quote von Antragsempfehlungen für Vorlagen der Sozialdemokraten gezeigt hatte. ${ }^{54} \mathrm{Im}$ Gegensatz zu den SPD-Politikern hielten sich die bündnisgrünen Abgeordneten bei Verfahren, zu denen ihre Fraktion keine Vorlage eingereicht hatte, in der Diskussion zurück und gaben lediglich ihren Bedenken gegenüber den vorliegenden Entwürfen Ausdruck. Eine Moderatorenrolle, wie sie die SPD in einigen Gesetzgebungsprozessen entwickelt hatte, war für die Bündnisgrünen nicht feststellbar.

\begin{tabular}{|l|l|l|l|}
\hline Tabelle 5: Strategische Präferenzen der Oppositionsfraktionen im Bayerischen Landtag \\
1994 bis 1998
\end{tabular}

54 Vgl. Katrin Steinack, a.a.O. (Fn. 11), S. 132 ff. 


\subsection{Erklärungsvariablen für die parteipolitischen Unterschiede}

Das gegensätzliche Auftreten der Fraktionen und die damit korrespondierenden parteispezifischen Strategien oppositioneller Einflussnahme können auf differierende Politikkulturen von SPD und Bündnis 90/Die Grünen zurückgeführt werden (vgl. Tabelle 6).

Die sachpolitische Orientierung der SPD-Fraktion und die machtpolitische Ausrichtung der Bündnisgrünen lassen sich durch ein Bündel von historisch-programmatischen Gründen erklären: Die Sozialdemokraten konnten zu Beginn der 13. Legislaturperiode auf eine fast 40-jährige Oppositionsvergangenheit im Bayerischen Landtag zurückblicken; keiner ihrer Abgeordneten hatte in seiner bisherigen politischen Karriere ein Regierungsamt bekleidet. Allerdings hatten viele der sozialdemokratischen Volksvertreter im Vorfeld ihrer parlamentarischen Laufbahn ein kommunales Wahlamt als Gemeinderat, Bürgermeister oder Kreisrat innegehabt. Die Erfahrung, dass sich im kommunalen Bereich vieles nur zusammen mit der CSU bewältigen lässt, hatte bei den Abgeordneten offensichtlich die Bereitschaft gefördert, Kompromisse einzugehen und sich pragmatisch für konsensuale Alternativen zu entscheiden. Dieser Bezug der Sozialdemokraten zur Realpolitik und die daraus resultierende Erkenntnis, dass sich nicht alles umsetzen lässt, was programmatisch erstrebenswert wäre, war auch in den Interviews hervorgehoben worden. 55

Das stärker sachpolitische Agieren der SPD-Vertreter ist zudem durch ihre direkte Konkurrenz zur CSU im Wettbewerb um die Wählerstimmen der politischen Mitte erklärbar. Die relativ ähnliche Positionierung erschwert das Besetzen von Extrempositionen sowie die Forderung nach Maximalzielen, zwingt stattdessen zu einer Orientierung an Machbarem und fördert damit die sachpolitische Auseinandersetzung mit der Regierungsmehrheit.

Im Gegensatz zur SPD, die ihre Wurzeln in der Arbeiterbewegung der Kaiserzeit hat, blicken die bayerischen Grünen auf eine kurze Parteigeschichte zurück. Ihre Ursprünge in der Bürgerbewegung und der außerparlamentarischen Opposition haben die Partei auch in ihren ersten parlamentarischen Jahren geprägt. Zwar ist das grüne Ideal, als „Antipartei“ vorrangig Kontrolleur des bestehenden politischen Systems zu sein, mit ihrer zunehmenden Parlamentarisierung verblasst. Anders als bei den Volksparteien erwarteten die Wähler allerdings mindestens bis zur ersten Regierungsbeteiligung der Grünen auf Bundesebene ausgeprägt linke Positionen. Den bayerischen Grünen dürfte es dabei gerade im Bereich der Sicherheits- und Innenpolitik, aber auch in der Agrar- und Umweltpolitik leicht gefallen sein, sich gegenüber der CSU sehr deutlich zu positionieren. Dass in Bezug auf die Umsetzung der programmatischen Vorstellungen mitunter Differenzen zwischen dem „Fundi“und dem „Realoflügel“ auftraten, wurde dabei nicht als hinderlich für die parteipolitische Profilierung gesehen, sondern als gegeben hingenommen beziehungsweise von den Wählern sogar antizipiert. ${ }^{56}$ Auch wenn sich der Auftritt der bündnisgrünen Abgeordneten im Plenum mittlerweile dem der Volksparteien angepasst hat, erachteten viele von ihnen auch in der 13. Legislaturperiode den öffentlichkeitswirksamen außerparlamentarischen Protest als probates Mittel, um politische Änderungen zu erreichen. ${ }^{57}$

55 Vgl. Interview 10-160201 CSU, Z. 263 f.

56 Vgl. Joachim Raschke, Die Grünen. Wie sie wurden, was sie sind, Frankfurt am Main 1993, S. 203, dem zu Folge die Existenz verschiedener Strömungen parteiintern als Ausdruck einer plural-heterogenen Partei und Merkmal innerparteilicher Demokratie bewertet wurde.

57 So wurde beispielsweise der bündnisgrünen Abgeordneten Irene Sturm von Fraktionskollegen vor- 
Das unterschiedliche Auftreten der beiden Fraktionen lässt sich zudem auf strukturelle Gründe zurückführen (vgl. Tabelle 6). Eine wesentliche Ursache für das sachpolitisch orientierte Handeln der Sozialdemokraten dürfte ihre größere Zahl an Abgeordneten sein: Weil sie in der 13. Legislaturperiode pro Ausschuss mindestens sechs Parlamentarier entsenden konnte, war es der Fraktion möglich, zu sehr vielen der im Landtag diskutierten Themen

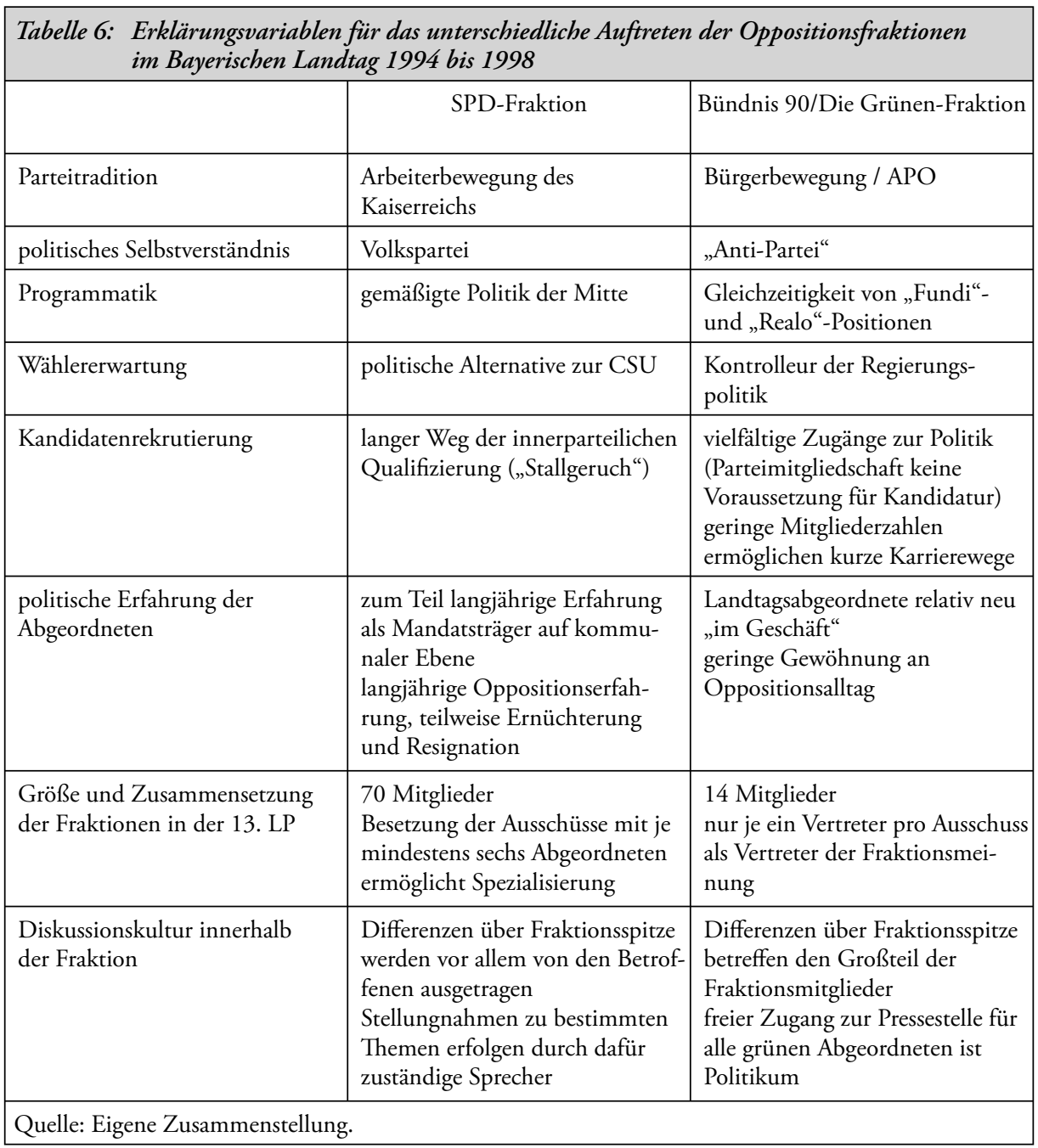

geworfen, dem außerparlamentarischen Protest gegen die Einlagerung von Castoren einen höheren Stellenwert zuzumessen als der inhaltlichen Arbeit in der Fraktion. Vgl. „Hier fehlt jegliche Perspektive zur Zusammenarbeit“. Bei den Grünen hängt der Haussegen schief, in: Süddeutsche Zeitung vom 11. Mai 1996. Aber auch Anträge, die von der Fraktion zum Thema BSE gestellt wurden, wurden beispielsweise durch die Blockade eines Schlachthofes durch Fraktionsmitglieder unterstrichen. 
Experten auszubilden, die ihre Sachkenntnisse entsprechend in die Ausschussdiskussionen einbrachten. Gleichzeitig machte die Vielzahl an Beteiligten aufwendige Abstimmungsprozesse innerhalb der Fraktion notwendig. Dabei ließen Aussagen interviewter SPD-Abgeordneter erkennen, dass dieser Ausgleich zwischen unterschiedlichen Interessenlagen zu einer starken Verwässerung politischer Themen führen und eine eindeutige Profilierung erschweren kann..$^{58}$

Im Gegensatz dazu entfielen für die 14-köpfige bündnisgrüne Fraktion aufwendige Abstimmungsprozesse. Dem jeweiligen Ausschussmitglied oblag es, exklusiv die Fraktionsmeinung zu vertreten. Dies erlaubte es den Bündnisgrünen, zumeist pointierter zu argumentieren und zu agieren als die sozialdemokratischen Kollegen. Aber auch die Diskussionskultur innerhalb der Partei und Fraktion war ihrem stärker machtpolitischen Auftreten förderlich: Bereits kurz nach Beginn der 13. Legislaturperiode trugen die Mitglieder der bündnisgrünen Landtagsfraktion ihre differierenden Ansichten über die „richtige“ Oppositionspolitik in langwierigen Flügelkämpfen offen zur Schau. Dass sich die Auseinandersetzungen gerade in der Frage niederschlugen, wie der Zugang zur Pressestelle geregelt werden sollte, deutet auf das Bestreben der Abgeordneten hin, generell stark in der Öffentlichkeit zu agieren und über diese zu kommunizieren. ${ }^{59}$

Der machtpolitische Vorsprung der bündnisgrünen Fraktion geht offensichtlich mit einem geringeren Engagement auf der sachpolitischen Ebene einher. Die Interviews mit den Abgeordneten zeigen zwar, dass es den Landtagsgrünen mittels einiger fachlich kompetenter Einzelpersonen gelungen ist (fraktionsübergreifend wurde vor allem der Bereich Haushalt genannt), an Kontur zu gewinnen. Politische Erfolge waren hier jedoch selten. Im Rahmen der untersuchten Verfahren ist eine von der Öffentlichkeit weitgehend unbemerkte sachpolitische Einflussnahme für die Bündnisgrünen nicht nachweisbar. Eine zentrale Ursache dürfte ihre geringe Abgeordnetenzahl und die daraus resultierende Beschränkung auf jeweils einen einzigen Ausschussvertreter sein. Aus der Spannbreite der dort behandelten Themen konnten die wenigen Parlamentarier lediglich einige vorrangige Punkte intensiv bearbeiten. Dabei konzentrierten sie sich auf Bereiche, die eine starke Resonanz in der Öffentlichkeit fanden und dazu beitragen konnten, ihr Profil als schlagkräftige Oppositionspartei zu schärfen. Für eher randständige Spezialgebiete, zum Beispiel den Immissionsschutz, konnte die Fraktion schon aus Gründen mangelnder Kapazität keine Experten ausbilden.

\section{Opposition im Bayerischen Landtag: nicht ohne Einfluss, aber strukturell benachteiligt}

Die zu Beginn zitierte These Gleitmanns, der Opposition im Bayerischen Landtag komme in der Gesetzgebung eine völlig belanglose Rolle $\mathrm{zu}^{60}$, kann angesichts der Untersuchungsergebnisse für die 13. Legislaturperiode nicht aufrechterhalten werden. Auch wenn SPD

58 Vgl. zum Beispiel Interview 21-151001 SPD, Z. 315.

59 Tatsächlich war die ungehinderte Nutzung der Pressestelle durch alle Parteiflügel einer der Hauptstreitpunkte, an denen sich die grünen Fraktionsquerelen zum Jahresbeginn 1996 entzündeten. Vgl. dazu Katrin Steinack, a.a.O. (Fn. 11), S. 81 ff. sowie: Fraktionschef und Pressesprecher beziehen Prügel. Die Landtags-Grünen spucken Gift und Galle, in: Süddeutsche Zeitung vom 4. Oktober 1996.

60 Vgl. Samy Gleitmann, a.a.O. (Fn. 10), S. 137. 
und Bündnis 90/Die Grünen keine eigenen Initiativen durchsetzen konnten, so ist es ihnen mit ihren Vorlagen dennoch gelungen, die CSU zum Handeln zu animieren und somit Bewegung in die Landespolitik zu bringen. Dabei bestätigen die von der Opposition gewählten Mittel und Wege der Einflussnahme das in der Forschung vorherrschende Bild einer thematisch bedingten Mischung von Konkurrenz und Kooperation im Umgang mit Vorlagen der Regierungsmehrheit. ${ }^{61}$

Gleichzeitig waren sowohl die von der SPD präferierte Strategie der sachpolitischen Kooperation als auch die von den Bündnisgrünen bevorzugte machtpolitische Auseinandersetzung erfolgversprechend. So konnte die Mehrheitsfraktion auch ohne die Einbindung der Öffentlichkeit zur Vorlage eigener Initiativen bewegt werden und war gut begründeten sachpolitischen Argumenten zugänglich. Ebenso zeigt der Erfolg einiger machtpolitischer Verfahren, dass die Opposition auch dann inhaltliche Veränderungen an Regierungsentwürfen erzielen konnte, wenn es sich (zum Beispiel bei der Gleichstellung der Geschlechter im Öffentlichen Dienst) um ein hochpolitisches Thema handelte, dem in der Öffentlichkeit hohe Aufmerksamkeit zukommt, und bei dem zu vermuten ist, dass die Regierungsmehrheit nur ungern Zugeständnisse macht. Zu bedenken ist bei dieser positiven Bewertung allerdings, dass SPD und Bündnisgrüne gegenüber CSU-Mehrheit und Staatsregierung nur sehr marginale qualitative Änderungen erreichten. Dies galt sogar dann, wenn (wie beim Kommunalwahlrecht für EU-Ausländer) eine hohe Übereinstimmung zwischen den oppositionellen Forderungen und übergeordneten Vorgaben bestand oder (wie bei der Kennzeichnung gentechnisch veränderter Lebensmittel) sich eine breite außerparlamentarische Allianz gegen den Mehrheitsentwurf formierte. Ungeachtet des öffentlichen Protests bestimmte letztendlich die Hegemonialmacht CSU, was konkret umgesetzt wurde.

Das Vorgehen von SPD und Bündnis 90/Die Grünen beweist, dass beide Fraktionen mit der Klaviatur oppositioneller Einflussmöglichkeiten umzugehen wussten. Selbst wenn das Themenspektrum der untersuchten Fälle nicht an die Fülle von Gebieten heranreicht, zu denen auf Bundesebene Gesetze verabschiedet wurden, und auch wenn die erzielte Presseresonanz nur sehr selten über Bayern hinaus reichte, konnte die Opposition demonstrieren, dass sie in ihrem Vorgehen ähnlich agil ist wie die parlamentarische Minderheit auf Bundesebene. Die kleineren Landtagsfraktionen entkräften damit den Vorwurf, in den Landesparlamenten laufe oppositioneller Protest automatisch ins Leere, da dort nur minder wichtige Materien verhandelt würden. ${ }^{62}$

Inwieweit es sich bei der eher konfrontativen Strategie von Bündnis 90/Die Grünen um ein typisches Merkmal kleiner und personell schlecht ausgestatteter Oppositionsparteien handelt, während größere Oppositionsfraktionen eher auf eine Politik der Kooperation setzen, muss in weiteren Untersuchungen geklärt werden. Denkbar wäre auch, dass es vornehmlich parteispezifische Besonderheiten sind, welche die jeweilige Oppositionspolitik und deren Wahrnehmung durch die Öffentlichkeit prägen. Die Klärung dieser Frage dürfte vor allem für die bayerische SPD als notorische Verliererin der Landtagswahlen von Bedeutung sein. Nach wie vor ist nämlich unklar, ob die Bayern-SPD innerhalb der sozialdemokratischen Landesverbände ein besonderes Profil aufweist, auf das ihre Erfolglosigkeit

61 Vgl. Ludger Gruber, Die CDU-Landtagsfraktion in Nordrhein-Westfalen 1946-1980. Eine parlamentshistorische Untersuchung, Düsseldorf 1998, S. 281.

62 Vgl. Manfred Friedrich, Parlamentarische Opposition in den deutschen Bundesländern, in: Walter Euchner (Hrsg.), a.a.O. (Fn. 18), S. 76 - 83, S. 77. 
zurückgeführt werden kann, oder ob vielmehr die strukturelle Benachteiligung des SPDLandesverbands gegenüber der Bundespartei CSU für die fortwährenden Niederlagen verantwortlich ist.

Zwar konnte die Untersuchung zeigen, dass die Oppositionsfraktionen im Bayerischen Landtag ihre Alternativ- und Kritikfunktion gegenüber der Mehrheitsfraktion aktiv wahrnahmen. Vordergründig lässt sich Gleitmanns These, ,gerade das für das Funktionieren einer parlamentarischen Demokratie so wichtige Wechselspiel zwischen Regierung, Mehrheitsfraktion und Opposition scheint in Bayern nicht zu funktionieren "63, damit als unbegründet ablehnen. Allerdings ist angesichts der engen Grenzen, in denen oppositioneller Erfolg möglich ist, eine Relativierung ihrer Einflussmöglichkeiten nötig. Die Benachteiligung gegenüber der Bundespartei CSU wird von den oppositionellen Landesverbänden als so eklatant empfunden, dass einige bayerische Sozialdemokraten die Forderung nach einer von der Bundespartei unabhängigen Bayern-SPD erheben. ${ }^{64}$ Während in anderen Bundesländern (zum Beispiel in Hamburg, im Saarland oder in Rheinland-Pfalz) die langjährige Vormachtstellung einer Partei durch abrupte Regierungswechsel beendet wurde, scheint dies für Bayern angesichts der weit unterdurchschnittlichen Wahlergebnisse der SPD nicht vorstellbar. Da die CSU bei der Landtagswahl 2003 über zwei Drittel der Landtagsmandate errungen hat, hat sich die Situation für die Sozialdemokraten vielmehr weiter verschärft. ${ }^{65}$

Die (durch die Parlamentsverkleinerung zusätzlich reduzierte) geringere Zahl an Abgeordneten erschwert die inhaltliche Vorbereitung auf die im Landtag verhandelten Themen und deren Vertretung im Ausschuss. Zudem beeinträchtigt sie die ohnehin schon relativ schlechte Repräsentanz von bündnisgrünen und SPD-Abgeordneten vor Ort. Die aus Wettbewerbsgründen notwendige stärkere Vernetzung der Parteien in Vorfeldorganisationen ist damit kaum zu leisten. Außerdem werden die in der Geschäftsordnung des Landtages festgelegten Kontrollmöglichkeiten der Opposition durch ihre geringe Größe reduziert. Besonders schwer dürfte für die beiden Fraktionen dabei wiegen, dass gerade die Untersuchungsausschüsse, die bislang eines ihrer stärksten Instrumente zur Kontrolle der Staatsregierung waren, mit einem Votum von zwei Dritteln ihrer Mitglieder den Ausschluss der Öffentlichkeit beschließen können. ${ }^{66}$ Der wichtige Gang an die Öffentlichkeit kann den Oppositionsfraktionen somit seit 2003 von der Mehrheit versperrt werden. Insgesamt ist damit eine erhebliche Verschlechterung oppositioneller Einflussmöglichkeiten eingetreten. Ob die Auseinandersetzung der oppositionellen Landesverbände mit der in Bayern anscheinend übermächtigen Bundespartei CSU vor diesem Hintergrund langfristig zum Regierungswechsel führen wird, bleibt abzuwarten.

63 Samy Gleitmann, a.a.O. (Fn. 10), S. 137.

64 Vgl. Brüder zur Sonne, zur SPB. Eine alte Forderung bekommt neue Anhänger: Die bayerischen [sic!] SPD soll sich von der Bundespartei lösen, in: Süddeutsche Zeitung vom 23. November 2003.

65 Zum Gewinn verfahrenstechnischer Machtmittel für die CSU-Fraktion durch die neuen Mehrheitsverhältnisse vgl. Günter Rieger, a.a.O. (Fn. 7), Fn. 66.

66 Vgl. Art. 25 BV sowie Anlage 3 zur GeschO BayLT. 